\title{
A Simple and Fast Method to Design Robotic Hands
}

\author{
Atefeh Hasan-Zadeh ${ }^{1, *}$, Osameh Irandoust ${ }^{2}$, Maryam Fayaz ${ }^{2}$, Asiyeh Sedghi ${ }^{2}$, Alireza Fateminiya ${ }^{2}$, Aref \\ Gheragali $^{2}$, Mahdiyeh Rahban ${ }^{2}$, Arman Karami ${ }^{2}$, Mohammadreza Nazemzadeh ${ }^{3}$, Mehrangiz Rostami ${ }^{4}$, Mehdi \\ Raeisosadat $^{4}$ \\ ${ }^{1, *}$ Assistant Professor, Fouman Faculty of Engineering, College of Engineering, University of Tehran, Iran, P.O. Box: 43581- \\ 39115 \\ ${ }^{2}$ BSc Student of Computer Engineering, Fouman Faculty of Engineering, College of Engineering, University of Tehran, Iran \\ ${ }^{3}$ BSc Student of Mechanical Engineering, Caspian Faculty of Engineering, College of Engineering, University of Tehran, Iran \\ ${ }^{4}$ BSc Student of Chemical Engineering, Fouman Faculty of Engineering, College of Engineering, University of Tehran, Iran
}

\begin{abstract}
This paper has investigated the mathematical approaches used in the design of robots in order to identify the best method to design robotic hands. For this purpose and following the main origin of the subject, the results obtained from the biomechanics of the legs of different families of insects have been collected. Geometric measurements of various pairs of legs designed for walking, running, swimming, digging and other related tasks have been presented from an entomological point of view and their relationship to the Fibonacci sequence. With the application of the optimization methods, the results obtained from observing the relationships between the design of organs (legs) of different families of insects with multi-joint structures and different mechanical abilities of the legs have been presented. These studies could be a natural inspiration for the development of multifunctional robotic arm technology. In the following, studies on the mechanical design of 4 configurations of robotic compliant fingers based on a compliant mechanism have been examined and analyzed to reduce costs and control complexity. Geometric parameters of compliant finger design as well as model prediction are performed by the Fibonacci sequence, which in addition to providing natural behavior, will be faster and easier than optimization methods. The design provided from the cited sources will be a suitable response to meet the needs of commercial prostheses and robotic arms.
\end{abstract}

Keywords: Fibonacci sequence, Foot biomechanics, compliant mechanism, Prosthetic finger, Robotic finger.

\section{INTRODUCTION}

In this paper, studies on non-dimensional relationships between different parts of insect legs have been analysed, [1-4]. The correspondence between the design of the organs (legs) of different families of insects with multi-joint structures and different mechanical abilities of the legs has been studied. Undoubtedly, such research could be a natural inspiration for the development of multi-purpose robotic arm technology.

In the following, we will investigate the artificial hands among the studies done in the field of robotics. Many research efforts have been made on artificial multi-fingered hand for specific applications, including prostheses, humanoid robots, and industrial robots (as industrial tools in factories, surgical assistants in hospitals, and in-house services and, of course, on the battlefield). Prostheses are another application for multi-fingered robots.

In the past, multi-fingered robotic hands attempted to mimic one or more of the specifications of a human hand. In recent years, a variety of significant architectures for robotic hands have been developed, including Barret Hand, Manus Colobi, LMS Hand, TBM Hand, DLR II Hand, Tuat/Karlsruhe Hand, BUAA Hand, GIFU II Hand, Hand RTR, Standford JPL Hand, Torino Hand, MA-I Hand, SARAH Hand, MIT Hand and RCH-I Hand. Most of these robotic hands have a high degree of freedom and therefore have a complex control system with increasing degrees of freedom and joints. In addition, their architecture has high costs. Considering these limitations, the interest in designing humanoid robots with simpler design and control has become a necessity. The term anthropomorphic refers to the ability of a robotic responder to mimic a human hand, in whole or in part, as well as its shape, size, coherence, and totality (including color, temperature, etc.). 
The major problem with the prosthetic hands mentioned earlier is the complexity of the mechanical design of the finger, their joints, and their built-in transfer mechanism. Gifue offered a distinctive link mechanism with 4 joints and 4 degrees of freedom, [5]. Almost all previous designs of robotic fingers had 3 degrees of freedom and 3 joints. The structure of the finger included conventional mechanisms and many parts or elements, [6-7].

One way to overcome this complexity is the commercial prosthetic hands, which have a simple structure. But they do not have the flexibility and skill to take a wide range of objects of different sizes and shapes and, heavier prostheses will make it more difficult to place the device and will have 2 or 3 joints. A conservative approach is a technique by which the complexity of control over a limited number of stimuli involving multiple fingers or a joint can be overcome. For this purpose, Carrozza offered a limited manual design with 8 degrees of freedom, which is driven by a single engine, [8]. In fact, the prosthetic hand has 3 joints with an activating motor, while other joints can only bend involuntarily. Another way to overcome the cost of production is the compliance mechanism to reduce the number of parts and the mechanical complexity of the multi-fingered hand, [7-9].

The compliant mechanism uses the elasticity of the material, which provides the flexuality of the hingers instead of the joints appearing in it, [10]. In this paper, the studies performed to design a compliant one-piece finger with 4 different configurations and the definition of their kinematics have been analyzed. Two joints with two degrees of freedom and three joints with three degrees of freedom are the main categories of designed fingers. Direct kinematics that provide the position and direction of the fingertip obtained according to numerical angles are the most important features of the compliant finger. In the research, the types of basic grasping have been classified based on their most appropriate use, and of course the geometric parameters of the 4 compliant finger designs follow the Fibonacci sequence.

\section{MATHEMATICAL STUDY OF INSECT LEGS: AN APPROACH TO ROBOTIC DESIGN}

\subsection{A natural comparison}

Lengths of the sections of 3 or 4 pairs of legs were measured using image measurement software depending on the insect family, (Image J 1.50i, National Institutes of Health USA (C), Fig. 1(A-B).

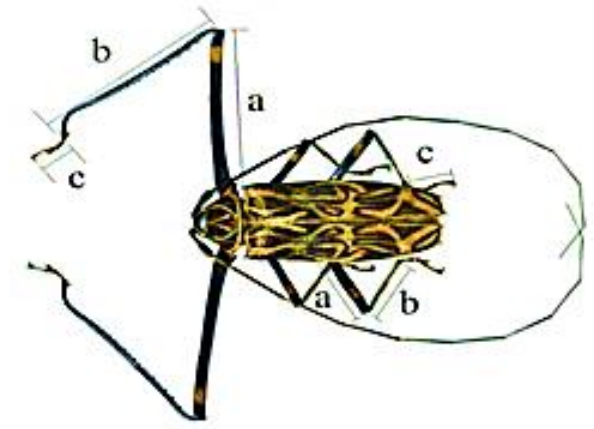

(A)

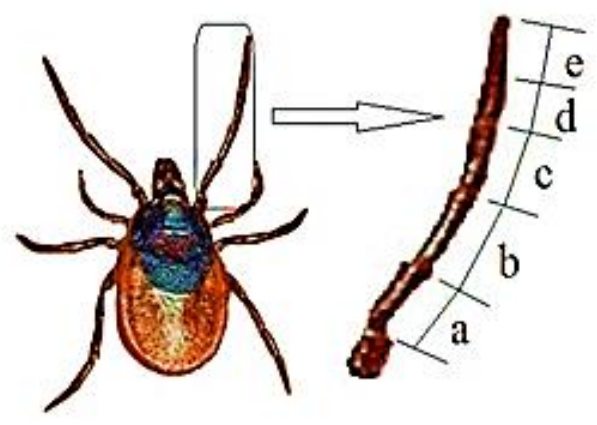

(B)

Fig. 1. Lengths of the sections of 3 or 4 pairs of legs, [4]

By studying 21 species of 20 insects in each group, the data of three consecutive sections of $s_{j-1}, s_{j}, s_{j+1}$ were measured from each leg and for numerical calculations of twice the following ratio has been used;

$$
W_{j}=\frac{\left(s_{j-1}+s_{j}\right)\left(s_{j}+s_{j+1}\right)}{s_{j}\left(s_{j-1}+s_{j}+s_{j+1}\right)}
$$

Thus, the leg with $\mathrm{n}$ joints is denoted by the $n-2$ value of $W$. The results of Equation (1) has been compared with twice the value of the ratio calculated for the Fibonacci sequence $\left\{F_{j}\right\}_{j=1}^{\infty}$ in Equation (2);

$$
W_{F}=\lim _{j \rightarrow \infty} \frac{\left(F_{j-1}+F_{j}\right)\left(F_{j}+F_{j+1}\right)}{F_{j}\left(F_{j-1}+F_{j}+F_{j+1}\right)} \approx 1.31
$$


The results have been obtained for different species as well as for each pair of legs with different functions separately. Insects that dig holes, hunt prey, and gather food have special legs in front of them; while the flying insects and some collectors have special pairs of legs in the back. In addition, the front/rear legs are used as an actuator arm to clean the eyes/wings.

\subsection{Results of comparison}

Some results on the species of insects and the different types of their legs have been indicated in Fig. 2. The average of the obtained parameters on each group has been calculated. The upper and lower straight lines indicate the golden mean ratio.

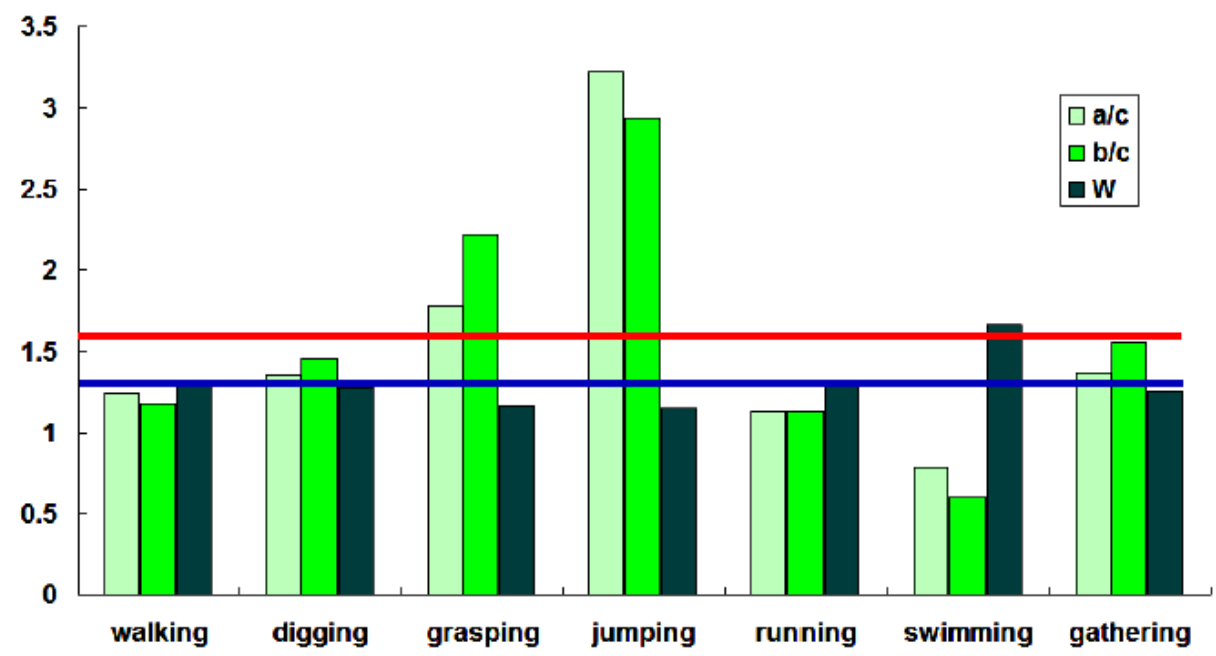

Fig. 2. Results on the species of insects and the different types of their legs, [4]

Also, unlike the arms and palms of humans and mammals, the adjacent parts of a-b-c (thigh and tibia or the fourth leg of the insect's leg and ankle) are not connected like the Fibonacci sequence. The relationship between a and $b$ and the relationship between $b$ and $c$ do not correspond to the golden ratio which is appropriate for many biomechanical systems. The only exception is the relationship between the tibia and the tarsus specific to the collection in bees. However, many legs are characterized by the relationship $W \approx W_{F}$.

The best (most optimal) mean for the $W$ benchmark for skill arms in mammals and humans is associated with running and walking legs (with standard deviation of $0.5 \%$ and $0.7 \%$, respectively). Exclusive digging and collecting legs have standard deviations of $2.8 \%$ and $4.4 \%$ that can be ignored. Grasping and jumping legs differ by $11 \%$ and $12.3 \%$ with the $W_{F}$ model, which is not a big difference for biological data. The swimmer's hands have a special design determined by the value of $W$, which is $27 \%$ more than the value of $W_{F}$. In most cases, the sections have been designed so that the leg can be fully bent into a chain or spiral, such as a 4-A chain or a 4-B spiral.

The lengths of the sections related to the measured data have been averaged for each specie. The momentum of the forces $m_{j}$ produced in the joints is reasonably proportional to the length $l_{j}$ of the corresponding parts. The bending dynamics of the leg are constant when it starts from the first a (shoulder) piece and reaches the shoulder at the end of the last c or e (tips of the claws) piece.

To apply the optimization benchmark, energy $E_{m}$ is considered for motion and $t_{0}$ is considered for operation time. Optimal Pareto solutions have been obtained for each case. The results obtained in Fig. 3 are shown in non-dimensional mode. According to the figure it can be seen that the relationship $l_{j-1} / l_{j}$ and $l_{j} / l_{j+1}$ in most cases with the value 62.38 is far away but close to the value of $W_{F}$.

Also, according to Fig. 3, walking and running legs have the best average in the benchmark test of $\left(E_{m}, t_{0}\right)$ legs. Digging legs have the slowest performance but are energy efficient. Jumping legs have the fastest performance but consume a lot of energy. In some areas, family boundaries are very close to each other, which means good performance for different types of activities such as digging, swimming, running, collecting and catching, Fig. 3. 


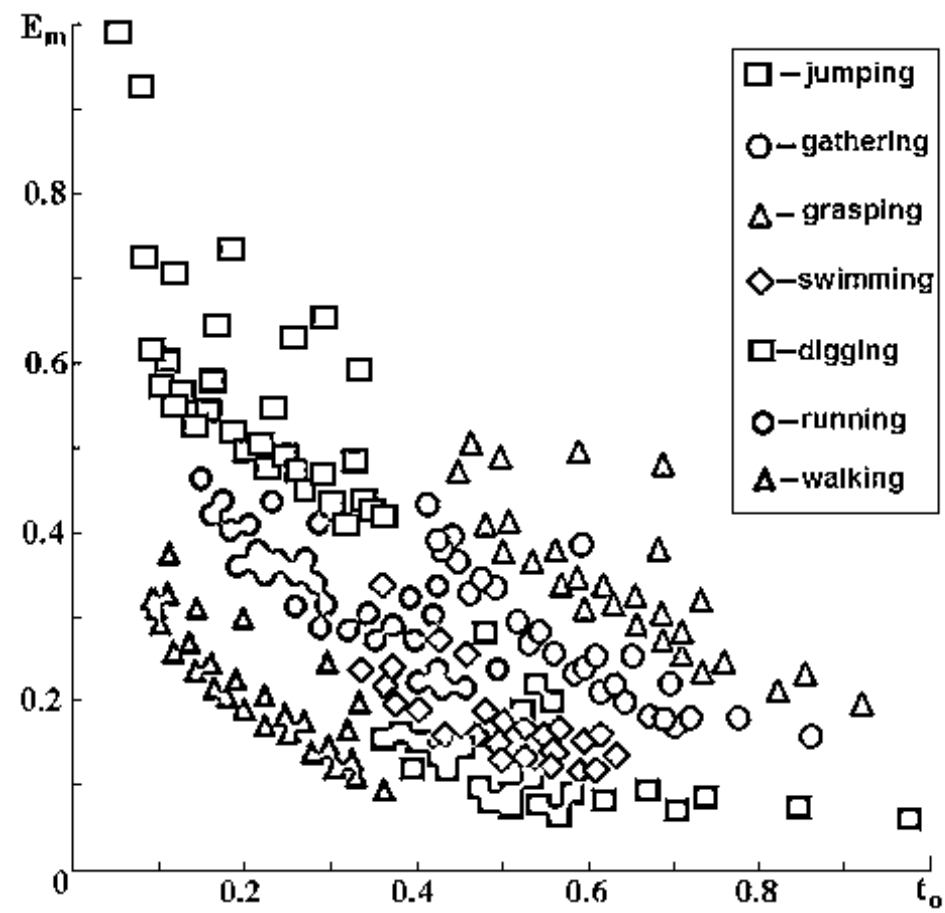

Fig. 3. Performance for different types of activities of legs, [4]

\section{MATHEMATICAL STUDY OF ARTIFICIAL FINGER IN PROSTHETICS AND ROBOTICS}

\subsection{Subject description}

The human hand is a complex system with 25 degrees of freedom that makes it difficult to copy its function and features. Distal interphalangeal or PIP, proximal interphalangeal or DIP, and metacarpophalangeal or MCP are natural joints of the hand of which the DIP and PIP must be flexible in one direction. The extra degree of freedom for the MCP joint causes the finger to move toward and away from the axis. Now, the studies performed to describe four different configurations of the compliant finger are investigated. They are classified in such a way that two of them have two degrees of freedom and two flexure hingers and the rest have 3 degrees of freedom and 3 flexure hingers. The finite element method, or FEM, is a tool for obtaining finger kinematics in any configuration.

\subsection{Description of the structure}

Fig. 4 shows four compliant finger designs.

First configuration: According to Fig. 4A, the structure consists of two rotating flexure hingers that mimic the natural DIP and PIP joints. Part A prevents lateral movement of the compliant finger during flexure and plays the same role for others. 


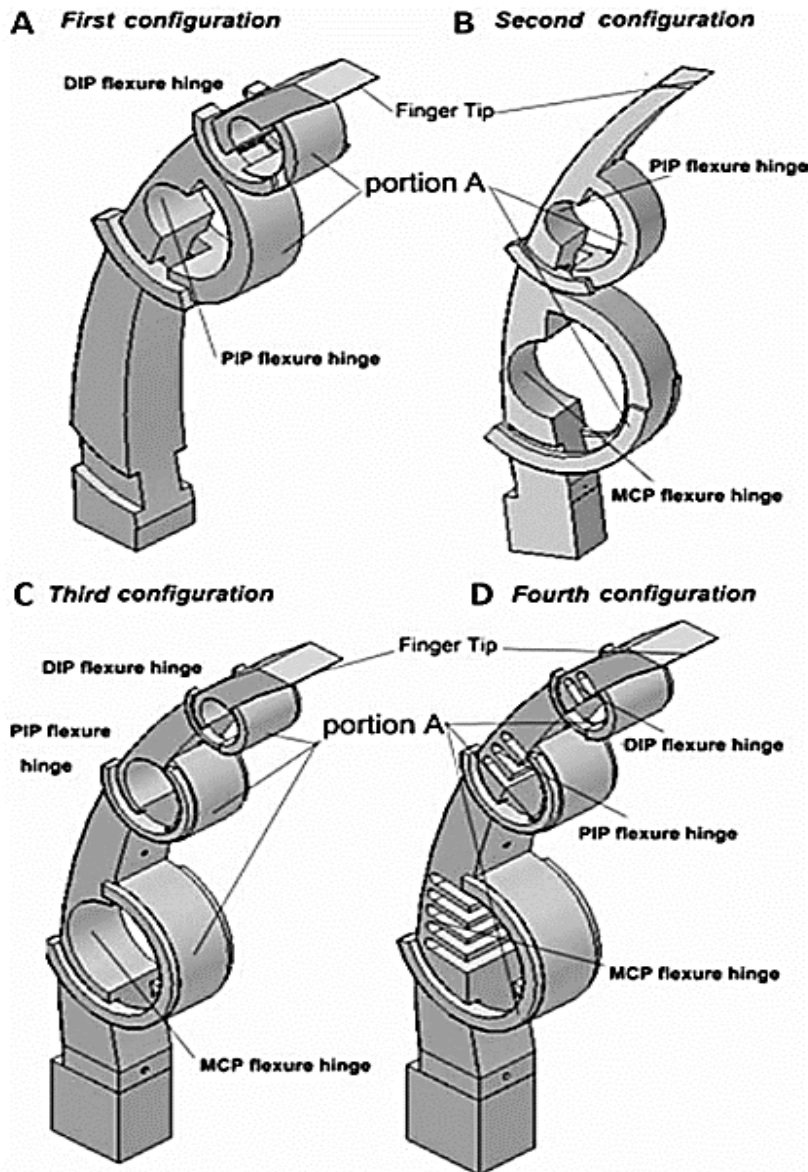

Fig. 4. Four compliant finger designs, [10]

The range of DIP and PIP flexure hingers is limited by the notch engraved in section A. Compliant finger joints represent asymmetrically rotating flexure hingers. Compliance or capacitance of its rotation about the axis $\mathrm{z}$ which is induced by the momentum in the direction $z$, i.e., $C_{1, \theta z-M z}$ with equations (3) and (4) is determined according to the following equations (3)-(4):

$$
\begin{gathered}
C_{1, y-M z}=\frac{12 r^{2}}{E b(r+t) \sqrt{t^{2}(2 r+t)^{5}}}\left(3 r^{2}+4 r+2 t^{2}\right) \sqrt{t(2 r+t)} \\
+3 r(r+t)^{2}\left(\tan ^{-1}\left(\left(\frac{r}{\sqrt{t(2 r+t)}}\right)+\frac{\pi}{2}\right)\right) \\
C_{1, \theta z-M z}=\frac{C_{1, y-M z}}{r}
\end{gathered}
$$

where, $C_{1, y-M z}, r, t, b$ and $E$, respectively, represent the compliance around the axis $y$ and the compliance induced by the momentum in the $z$ direction, radius, thickness and modulus of the material.

The radii of the rotating hingers as well as the width and length of the fingers or the bones of the compliant fingers follow the Fibonacci sequence. Fig. 5A shows the main geometric parameters related to the compliant finger design. 
A First configuration

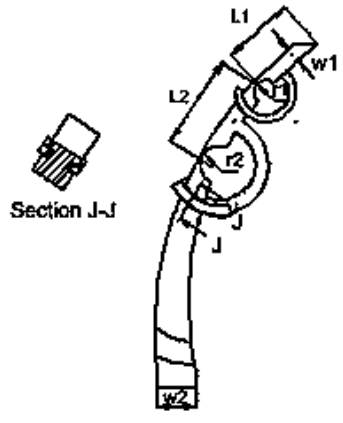

C Third configuration

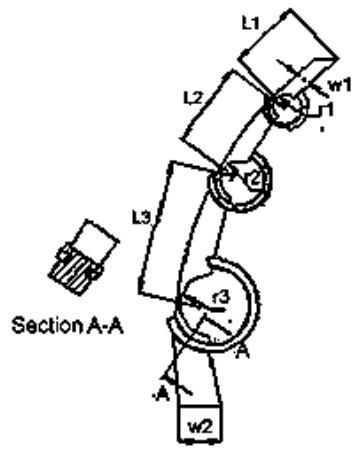

B Second configuration

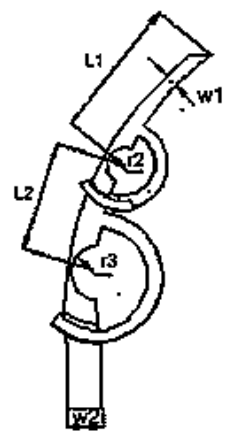

D Fourth configuration

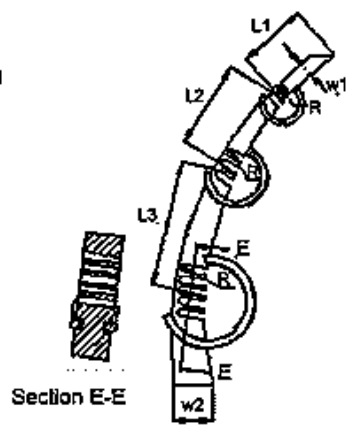

Fig. 5. Main geometric parameters related to the compliant finger design, [10]

$L_{1}$ and $L_{2}$ denote the length of each finger bone (DIP and PIP). The radii of the compliant rotating hingers are $r_{1}$ and $r_{2}$. Also, $W_{1}$ and $W_{2}$ represent the width of compliant finger for the tip and base of the finger, respectively, as shown in Fig. 5A.

The finger has been made of plastic. Part A, as shown in Fig. 4A, performs the main function of preventing lateral movements by compliant hingers. Each of the $L_{i}(i=1,2,3)$ compliant finger joints corresponds to the front, middle, and back bones of the human finger, respectively, and each joint in the $\theta_{i}$ coordinate frame $i(i=1,2,3)$ can be considered as MCP, PIP and DIP joints, respectively.

In the studies, the finite element method or FEM has been used to define compliant finger kinematics from the flexing position. The FEM model will use plastic materials with Yang modulus of $3 \mathrm{GPa}$ and a level of 183 as the type of element. The flexing angles of DIP, i.e. $\theta_{1}$ and PIP, i.e., $\theta_{2}$ of the compliant finger 1 have been shown in Fig. 6A in different positions. The general kinematics for the placement of the end or upper effector of the four compliant fingers depend on the flexure angles. 


\section{A compliant finger 1 B compliant finger 2 C compliant finger 3 D compliant finger 4}

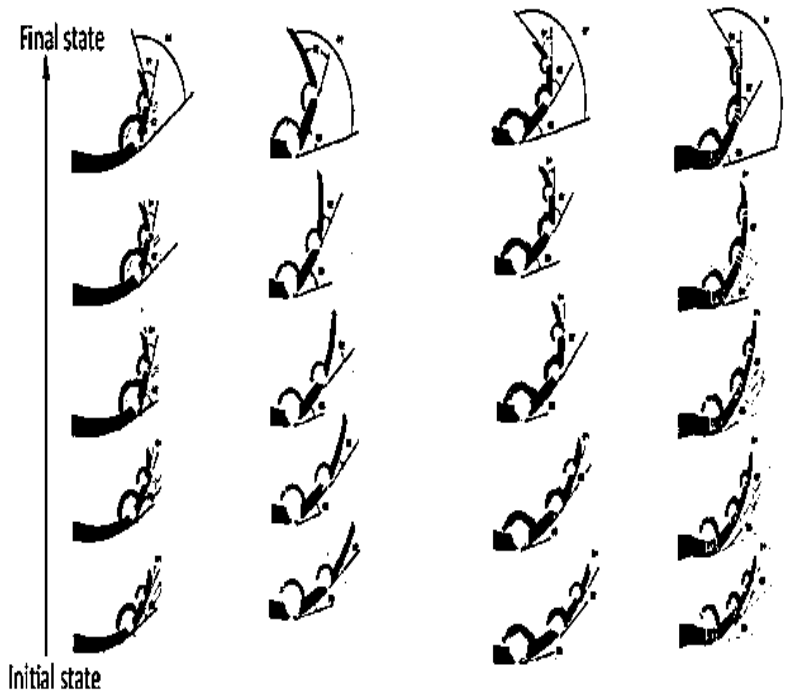

Fig. 6. Flexing angles of the compliant finger, [10]

Thus the relations of finger kinematics are presented by equations (5), (6) and (7);

$$
\begin{gathered}
x_{f}=L_{1} \cos \theta_{1}+L_{2} \cos \left(\theta_{1}+\theta_{2}\right)+L_{3} \cos \left(\theta_{1}+\theta_{2}+\theta_{3}\right) \\
y_{f}=L_{1} \sin \theta_{1}+L_{2} \sin \left(\theta_{1}+\theta_{2}\right)+L_{3} \sin \left(\theta_{1}+\theta_{2}+\theta_{3}\right) \\
\theta_{f}=\theta_{1}+\theta_{2}+\theta_{3}
\end{gathered}
$$

where, $x_{f}$ and $y_{f}$ represent the position of the fingertip in the direction of $x$ and $y$, respectively, and $\theta_{f}$ denotes the state (or rotation) of the tip of the finger.

Second configuration: The second configuration consists of 2 rotating flexure hingers that mimic natural PIP and MCP joints. The image of this configuration has been shown in Fig. 6B and has the type of flexure. Fig. 6B confirms the flexure of this configuration and the range of the PIP and MCP joints.

Third configuration: The third configuration consists of 3 rotating flexure hingers that mimic 3 natural hand joints and have 3 degrees of freedom. Fig. 6C shows this configuration. The main difference of this configuration with the previous type is in the joint added to the finger. The FEM simulation of this configuration through the flexing site has been indicated in Fig. 6C.

Fourth configuration: What distinguishes this design from the rest is its flexure hinger, which is made up of multiple asymmetric rotating hingers. The numbers 2, 3 and 5 represent the Fibonacci numbers as well as the number of hingers on each joint. Fig. 7D shows the distribution of compliant hingers in the finger. The new compliance of the hinger with the multiplication equation (3) is shown in which the number of hingers represents the fingers. FEM simulation illustrates the behavior of multiple hingers and flexure between them in three dimensions. 


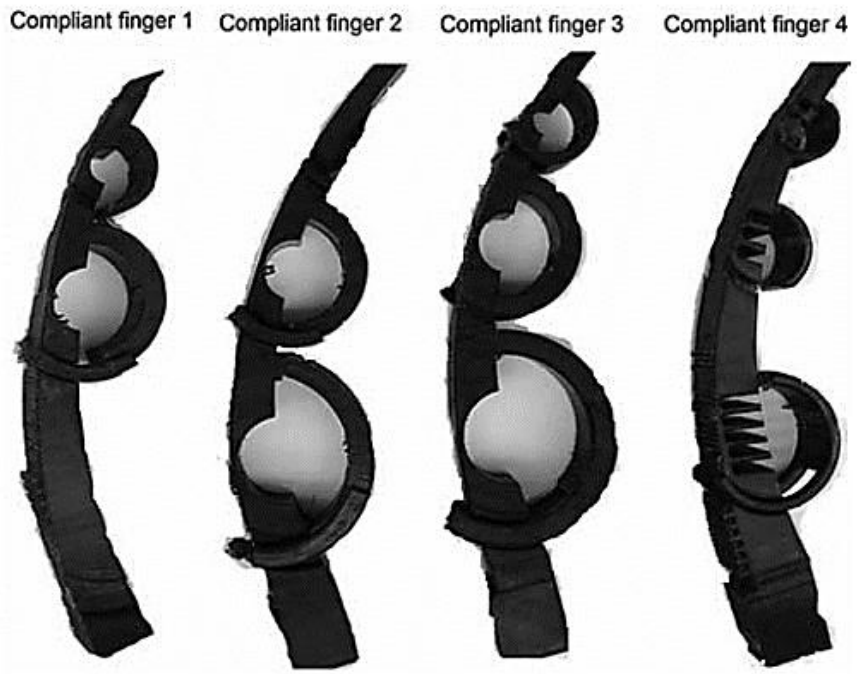

Fig. 7. Distribution of compliant hingers in the finger, [10]

\subsection{Prototype}

A desktop 3D printer (Marker Bot Replicator) has been used to fabricate the fingers using a polylactic acid string. The value of Yang modulus of this type of printed thermoplastic is $3-3.5 \mathrm{GPa}$ and the tensile strength of this specimen is $35-50 \mathrm{MPa}$ and the applied solvent is limonene. Fig. 7 shows the fingers made by the prototype made quickly. The structure of each finger weighs approximately 50 grams and the fabrication duration for a finger is 4 hours.

\subsection{Results obtained from compliant finger design}

Fig. 8 describes the flexural movements of a compliant finger joint. The joints DIP, $\theta_{1}$ and PIP, $\theta_{2}$ show the compliant finger 1 in an acceptable range of the finger tip rotation, $\theta_{f}$, as shown in Fig. 8. A.

The joints PIP, $\theta_{1}$ and MCP, $\theta_{2}$ show the compliant finger 2 through flexure in Fig. 8B. Fig. 8(C-D) show the flexure simulation of compliant 3-joint fingers. The range of motion of the PIP joints, $\theta_{2}$ of all compliant fingers, is larger than that of the DIP joints, $\theta_{1}$, as shown in Fig. 8. Fig. $8 \mathrm{~B}$ shows the MCP joint, $\theta_{3}$, which is smaller than the PIP, $\theta_{1}$. But the range of MCP joints, $\theta_{3}$, is large according to Fig. 8(C-D). These curves are described in Fig, 8 by fitting the data for the FEM results, as shown in Fig. 6.

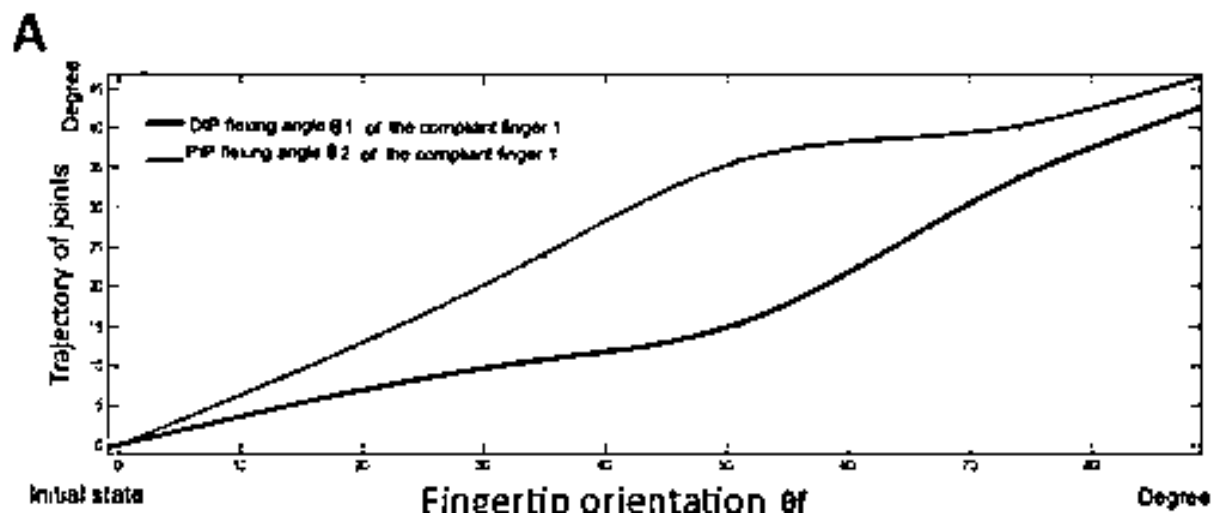



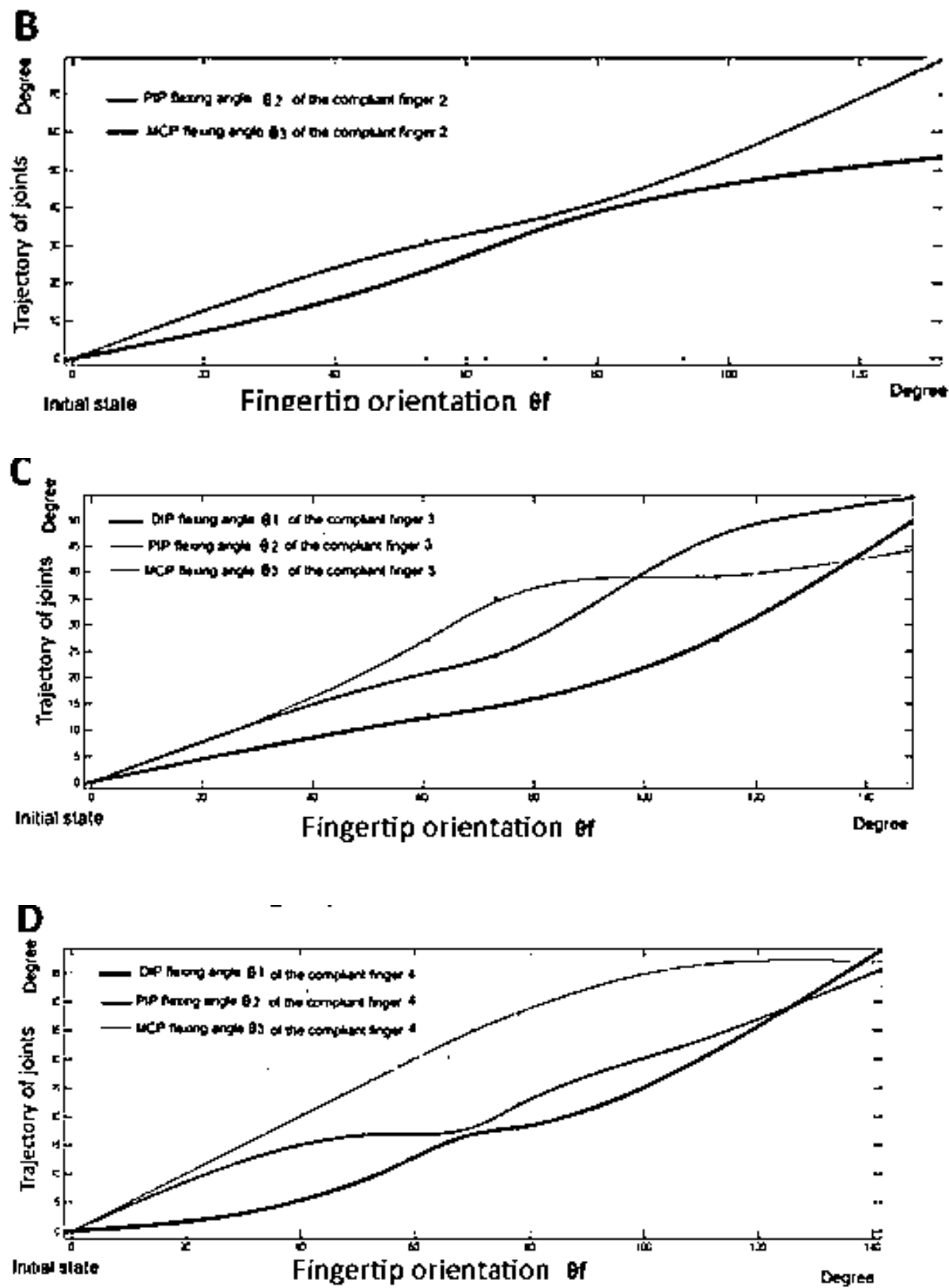

Fig. 8. Flexural movements of a compliant finger joint, [10]

\section{RESULTS}

In this paper, geometric studies performed on the examination of insect organs from a mechanical point of view were presented. The relationships obtained are useful for designing multipurpose arms and legs in robotics, because the design of robotic organs is based on an optimization criterion such as energy consumption, operating time and simplicity of control, which was considered in this study. Studies were also presented on four new finger designs compliant with a cost-effective element by considering the Fibonacci sequence for designing and predicting random variables.

The Fibonacci sequence, in addition to providing a natural look and feel, defines the finger design variable faster and easier than the optimization method. It is possible to use any finger designed for use in prosthetics and robotics due to its kinematics and range of motion and the new joint curvature design also reduces stresses on the compliant finger.

\section{REFERENCES}

[1] R. Dudley, The biomechanics of insect flight: form, function, evolution, Princeton: Princeton University Press, 2000.

[2] R. E. Ritzmann and S. N. Zill, Walking and jumping, in Encyclopedia of insects, 2009, Ed. by V. H. Resh, R. T. Cardé. San Diego: Academic Press, p. 1044-1048. 
[3] J. Casas, S. J Simpson, [eds.], Advances in insect physiology, vol. 34: insect mechanics and control, 2008, San Diego: Academic Press.

[4] O. Denisov and N. Kizilova, Geometry and mechanical function of multijoint extremities from mammals to insects: towards biomimetic design of robotic arm, 11th International Conference Biomdlore, Druskininkai, Lithuania, OCTOBER 20-22, 2016, p. 69-72.

[5] H. Kawasaki, T. Komatsu and K. Uchiyama, Dexterous Anthropomorphic Robot Hand with Distributed Tactile Sensor: Gifu Hand, IEEE/ASME Trans Mechatron, 2002, 7(3), p. 296-303.

[6] J. T. Belter, Performance Characteristic of Anthropomorphic Prosthetic Hands, IEEE Int Conf Rehabi Robot, 2011.

[7] M. A. El-Sheikh. M. F. Taher and S. M. Metwali, New optimum humanoid hand design for prosthetic applications, Int J Artif Organs, 2012, 35(4), p. 251-262.

[8] M. C. Carrozza, C. Suppo and F. Sebastiani, The Spring Hand: Development of a Self-Adaptive Prosthesis for Restoring Natural Grasping, Auton Robots, 2004, 16(2), p. 125-141.

[9] M. A. El-Sheikh. M. F. Taher, The design of a modular voluntary opening prosthetic hand: ongoing research and preliminary results. Int J Artif Organs, 2016, 39(5), p. 235-241.

[10] M. A. El-Sheikh, Fibonacci-Complaint finger design, Int J Artif Organs, 2016, 39(9), p. 491-496. 